\title{
Object-quality learning-set formation in the young chicken'
}

RODNEY J. PLOTNIK ${ }^{2}$ AND ROBERT B. TALLARICO

UNIVERSITY OF MIAMI
Four young chickens were given 50 object-quality learningset (LS) problems. Across problems the chickens showed increasing interproblem transfer or LS formation. The LS performance of the chickens matched some mammals-raccoon, cat, marmoset-in spite of the absence of a well developed cerebral cortex in the chicken.

There is evidence from interproblem learning or learning-set (LS) formation for an orderly phyletic trend toward greater learning capacity within the vertebrate series (Warren, 1965)。Nissen (1951) has suggested that some of the higher nonmammalian vertebrates may be behaviorally more advanced than certain of the lower mammals. Evidence for LS formation in birds (Alpert et al, 1962; Bacon et al, 1962) and Zeigler's (1961) work which equates LS performance of the chicken with the mammal (cat) supports Nissen's suggestion. However, comparison of the chicken's LS performance with other species is still tentative.

Because ability to form LS has been widely used as an index of an animal's learning capacity, this study investigated the LS capacity of the chicken, Class Aves, and compared LS performance of the chicken with mammals.

\section{Method}

The Ss were four White Plymouth Rock roosters, approximately 4 months old and weighing about 4 pounds . The Ss were maintained at $80 \%$ of stable body weight throughout experiment.

The apparatus was a rectangle 12 in. wide, 24 in. high, and $30 \mathrm{in.}$ long with two separated stimulus windows at one end. An illuminated, enclosed stimulus tray outside the windows contained two food wells covered with pivoting metal disks on which stimuli were placed. A guillotine door, inside the windows, determined when the Ss could view the stimuli. From pilot work it was noted that the $S$ regularly remained in front of and responded to the stimulus window last reinforced, thereby decreasing the chance to visually compare the two stimuli. To correct this perseverative tendency, the $\mathrm{S}$ was pushed $12 \mathrm{in}$. back from the stimulus windows by a manually moved pusher which consisted of a rod attached to a vertical board.

The stimuli were 50 three dimensional objects (toys, statuary, wood blocks) arranged into 50 pairs. The stimuli composing the pair were rotated according to the Gellerman series.

Before LS testing began, the Ss were pretrained for three days on the following trial procedure. The trial began when the guillotine door was opened, showing the stimuli. The pusher was quickly drawn forward, allowing the $S$ to approach the stimuli. If the $S$ put his head into the correct window, the stimulus disk pivoted, allowing $\mathrm{S}$ two pecks at pellets in food well beneath disk. If $\mathrm{S}$ chose incorrect window, he was pushed back, guillotine door was dropped and $10 \mathrm{sec}$. elapsed before next trial began. A total of 50 problems were given at the rate of 80 trials or 2 problems a day for the first 25 problems and 80 trials or 3 problems a day for the last 25 problems. Criterion was 8 correct responses out of 10 consecutive trials.

\section{Results}

One index of LS formation is the percentage change of correct responses on Trial 2. Figure 1 shows that across 50 problems there is an increase from $35 \%$ to $70 \%$ correct responses on Trial 2. This indicates that the chicken does show interproblem transfer on a series of objectquality discrimination problems. Looking at LS formation in terms of trials to criterion, problem 2 was solved in 46 trials, while problems 20 to 50 averaged 12 trials to criterion.

The Ss perseverated on both object and side. Both types of perseveration decrease with practice: stimulus perseveration decreased after problem 33, and side perseveration decreased after problem 39 .

From a number of different studies, Johnson \& Michels (1958) have drawn a composite figure showing the relative LS performance of several species. The data from this study were superimposed on their figure to compare the chicken with other species. Figure 2 shows that the LS performance of the chicken matches

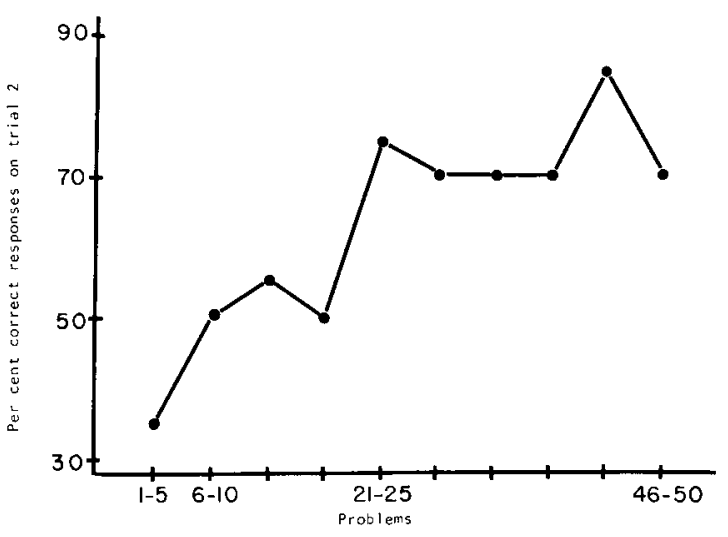

Fig. 1. Group data showing the per cent correct responses on trial 2 with problems in groups of 5 . 


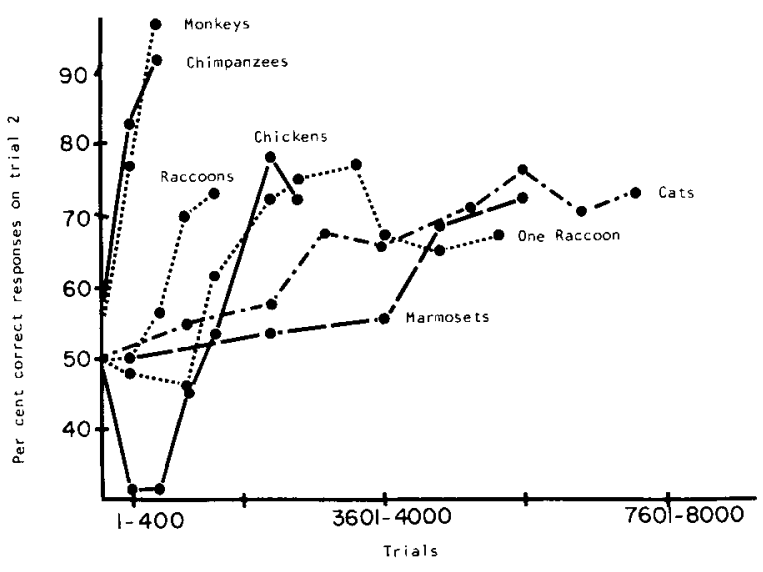

Fig. 2. Learning-set performance of chickens and other animals in terms of the percentage of correct responses on trial 2 of successive groups of problems and total trials elapsed. (Johnson \& Michels, 1956, p. 378)

the LS performance of the raccoon, cat and marmoset which appear to asymptote at about the same level. All the later are inferior in performance to the higher primates.

\section{Discussion}

The speed or facility of LS performance shown by an animal will vary with the kind of data analysis. Using trials to criterion as an index, the chicken's performance approximates that of the primate (Harlow, 1949); using percentage correct responses on Trial 2 as an index, the chicken's LS performance approximates that of the racoon, cat and marmoset. Response on Trial 2 appears to be the more sensitive index of LSability. Changing or remaining with a stimulus on Trial 2, after reinforcement on Trial 1, critically assess the degree of intraand interproblem transfer.
This study confirms the work of Alpert et al (1962), Bacon et al (1962), and Zeigler (1961) who also demonstrated LS formation in chickens. From these studies it is apparent that although anatomically the chicken lacks a well developed cerebral cortex compared to mammals, behaviorally the chicken's LSperformance is similar to that of some more cortically developed mammals.

The chicken, a member of Class Aves, is generally assigned a phylogenetic position below that of mammals. The present results which rank the chicken behaviorally equivalent to the raccoon, cat, and marmoset, support Nissen's suggestion that some of the higher nonmammalian vertebrates may be behaviorally more advanced than certain lower mammals.

\section{References}

Alpert, M., Schein, M. W., Beck, C. H., \& Warren, J. M. Learningset formation in young chickens. Amer. Zool., 1962, 5, 502, (Abstract).

Bacon, H. R., Warren, J. M., \& Schein, M. W. Non-spatial reversal learning in chickens. Anim. Behav., 1962, 10, 239-243.

Harlow, H. F. The formation of learning sets. Psychol. Rew., $1949,56,51-65$.

Johnson, J. I., \& Michels, K. M. Learning-sets and object size effects in visual discrimination learning by raccoons. $J$. comp. physiol. Psychol., 1958, 51, 376-379.

Nissen, H. Phylogenetic comparison. In S. S. Stevens (Ed.), Handbook of experimental psychology. New York: Wiley, 1951.

Warren, J. M. Primate learning in comparative perspective. In Allan M. Schrier, Harry F. Harlow, Fred Stollnitz (Eds.), Behavior of nonhuman primates. New York: Academic Press, 1965.

Zeigler, H. P. Learning-set formation in pigeons. J. comp. physiol. Psychol., 1961, 54, 252-255.

\section{Notes}

1. This investigation is based on a thesis submitted by the senior author in partial fulfillment of the requirements for the $M$. $S$. degree at the University of Miami.

2. Now at the Center for Neurobiological Sciences, University of Florida, Gainesville. 